\title{
Inhibitors of CAMP and CGMP phosphodiesterases enhance neurite outgrowth from cultured cortical neurons in a PKG dependent
} manner

\author{
Lida Kimmel, Mary Collins, Christopher J Schmidt, Frank Menniti and \\ Robin Kleiman*
}

Address: Neuroscience Discovery, Pfizer Global Research and Development, Groton, CT, USA

Email: Robin Kleiman* - robin.j.kleiman@pfizer.com

* Corresponding author

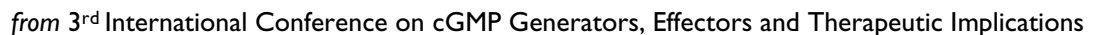

Dresden, Germany. I5-17 June 2007

Published: 25 July 2007

BMC Pharmacology 2007, 7(SuppI I):P33 doi:I0.1 186/I47I-2210-7-SI-P33

This abstract is available from: http://www.biomedcentral.com/I47I-22/0/7/SI/P33

(c) 2007 Kimmel et al; licensee BioMed Central Ltd.

Cyclic nucleotide signalling plays a key role in regulating neurite outgrowth in developmental and injury settings. The functional consequences of enhanced cyclic nucleotide signalling on processes that contribute to neurite outgrowth varies across neuronal cell type, age, cellular environment, and endpoints evaluated. We have evaluated the potential for neurite outgrowth in cultured embryonic cortical neurons in the presence and absence of selective inhibitors of many different phosphodiesterases. We observe differential enhanced outgrowth of several parameters of neurite outgrowth measured using the Array Scan automated image analysis platform. DT-2, a selective inhibitor of $\mathrm{PKG}$, dose dependently inhibited neurite outgrowth in this culture system and precluded PDE inhibitor mediated potentiation of neurite outgrowth parameters, including the enhancements produced by the CAMP PDE4 inhibitor, rolipram. By contrast, a selective inhibitor of PKA, PKI had no effect on neurite outgrowth in this culture system. These studies suggest that embryonic cortical neurons are critically dependent on PKG activity for neurite outgrowth. The finding that inhibitors of a CAMP PDE can facilitate neurite outgrowth in a PKG dependent fashion suggests that cAMP mediated enhancement of outgrowth still requires a PKG dependent step to express the potentiating effects on neurite outgrowth. 